\title{
Polarized Antiprotons-The Quest For A Missing Tool
}

\section{Erhard Steffens}

Citation: AIP Conference Proceedings 1149, 80 (2009); doi: 10.1063/1.3215768

View online: https://doi.org/10.1063/1.3215768

View Table of Contents: http://aip.scitation.org/toc/apc/1149/1

Published by the American Institute of Physics

\section{Conference Proceedings}

\section{Get $30 \%$ off all print proceedings!} Enter Promotion Code PDF30 at check 


\title{
Polarized Antiprotons - The Quest For A Missing Tool
}

\author{
Erhard Steffens \\ Physikalisches Institut, University of Erlangen-Nürnberg \\ Erwin-Rommel-Str. 1, D-91058 Erlangen, Germany \\ steffens@physik.uni-erlangen.de
}

\begin{abstract}
After termination of the LEAR facility in 1996 and the restriction of the CERN antiproton program to Trap experiments, stored antiprotons at low and medium energies are no longer available for experiments. FAIR at GSI (Darmstadt) will provide intense stored antiproton beams in less than a decade from now. This has renewed the interest in polarized antiprotons originally proposed for LEAR. In August 2007, an International Workshop was organized at the Cockcroft Institute (Daresbury) and methods to polarize stored antiprotons were discussed. In June 2008, a Heraeus Seminar at the Physikzentrum in Bad Honnef dealing with the same subject took place. The results of these workshops will be presented with some historical remarks and an account of the recent developments.
\end{abstract}

Keywords: Antiprotons, spin polarized beams, spin filtering.

PACS: $24.70 .+\mathrm{s} 25.43 .+\mathrm{t} 29.27 . \mathrm{Hj}$

\section{INTRODUCTION}

The $\bar{p} p$ interaction has been studied extensively using secondary antiproton beams at BNL and KEK, and by utilizing stored antiprotons at LEAR/CERN (1983-1996). Phenomenologically, the $\bar{p} p$ interaction at low momenta can be described by two potentials, the elastic one, $\mathrm{V}_{\mathrm{el}}$, and the annihilation potential $\mathrm{V}_{\mathrm{ann}}$. The elastic $\bar{p} p$ potential is related to $V_{\mathrm{el}}^{\mathrm{pp}}$ by the G-parity transformation. No analogue to $V_{\mathrm{ann}}$ exists in the pp channel. Experiments on the spin dependence in the $\bar{p} p$ system are restricted so far to single spin experiments, i.e. measurements of $\mathrm{A}_{0 \mathrm{n} 00}$ or $\mathrm{A}_{000 \mathrm{n}}$ [1]. The study of the $\bar{p} p$ spin correlation in the entrance channel requires beams of polarized antiprotons. Attempts at LEAR to produce polarized $\bar{p}^{\prime} s$ by double scattering were unsuccessful. The FILTEX proposal to polarize stored $\bar{p}^{\prime} s$ through Spin Filtering by means of a polarized internal hydrogen gas target was submitted in 1985 [2]. The development of this new method took too long to become part of the LEAR program which was terminated in 1996. Since then, the CERN $\bar{p}$ program is restricted to experiments at very low energies which are conducted at the CERN Antiproton Decelerator (AD).

In 2007, the new international FAIR project initiated by GSI (Darmstadt) was officially launched. FAIR stands for 'Facility for Antiproton and Ion Research' [3]. The completion is scheduled for 2016. A substantial fraction of the program is devoted to antiproton physics with multi-GeV beams at the High Energy Storage Ring

CP1149, Spin Physics, $18^{\text {th }}$ International Spin Physics Symposium

edited by D. G. Crabb, D. B. Day, S. Liuti, X. Zheng, M. Poelker, and Y. Prok

(C) 2009 American Institute of Physics 978-0-7354-0686-5/09/ $\$ 25.00$ 
(HESR). A general consensus exists that a new powerful $\bar{p}$ source should be complemented by a polarized antiproton beam. The PAX collaboration [4] has proposed to polarize $\bar{p}^{\prime} s$ in a small storage ring (APR) of suitable energy by means of Spin Filtering $[4,5]$. As key experiment the study of the double polarized Drell-Yan process $p \uparrow \bar{p} \uparrow \rightarrow e^{+} e^{-}$has been identified [6,7].

Since the first workshop at Bodega Bay in 1985, organized by O. Chamberlain and A. Krisch [8], several methods to polarize $\bar{p}^{\prime} s$ stored in a ring have been studied or proposed. In the following, previous attempts to develop an antiproton polarizer are discussed. In the wake of the PAX proposal, and 22 years after Bodega Bay, the next workshop on polarized $\bar{p}^{\prime} s$ was conducted in 2007 at the Cockcroft Institute (Daresbury) [9], followed by a Heraeus Seminar at the Physikzentrum (Bad Honnef) in 2008 [10]. The results of the two workshops are summarized. These and recent results obtained at COSY by the ANKE and PAX collaboration on depolarization of protons by co-moving electrons [11] represent impressive progress towards polarized $\bar{p}^{\prime} s$.

\section{HISTORY OF POLARIZED ANTIPROTONS ${ }^{1}$}

In the early 1980 's, the CERN $S p \bar{p} S$ collider came into operation leading to the discovery of the $Z^{0}$ and $\mathrm{W}^{ \pm}$bosons. Its powerful $\bar{p}$ source enabled a low energy $\bar{p}$ program at LEAR, a cooler and stretcher ring. First ideas by Kilian and Möhl appeared to polarize antiprotons by Spin Filtering. This process was first proposed by Shapiro [12] in 1966 to polarize thermal neutrons by spin-dependent attenuation in a polarized hydrogen target. It should be noted that spin filtering of slow neutrons by means of a polarized ${ }^{3} \mathrm{He}$ gas cell is now a standard method in neutron physics [13].

At the Bodega Bay workshop in 1985 [8], eleven potential methods to polarize stored $\bar{p}^{\prime} s$ were identified and discussed. Out of these eleven, two ideas were studied in depth afterwards: (i) The 'Spin Splitter' is based on repeated 'Stern-Gerlach kicks' resulting in a spatial separation of the two antiproton spin states. It has been discussed theoretically in a number of papers (e.g. [14]) and workshop contributions, but not yet been demonstrated experimentally. (ii) The Spin Filter proposal for LEAR [2] was actively pursued by the FILTEX collaboration. Polarization by Channeling through magnetized ferromagnetic foils was also discussed. Recently, the idea of Channeling by bent crystals as polarizer received some attention [9].

\section{The Filtex Test Experiment at the TSR (Heidelberg)}

The Filter Test was performed in the Heidelberg Test Storage Ring (TSR), a low energy ion storage ring with electron cooling and large acceptance [15]. The principle of the experiment is shown in Figure 1. Protons (or antiprotons in the final experiment) are stored in a ring with electron cooling. They traverse polarized hydrogen gas, confined in an open T-shaped storage cell fed by an Atomic Beam Source. The target polarization is oriented by means of a weak guide field vertically, parallel to the B-field in the ring dipoles, the stable spin direction. Low-B quadrupoles

\footnotetext{
${ }^{1}$ for more details see reference 9, p. 1
} 


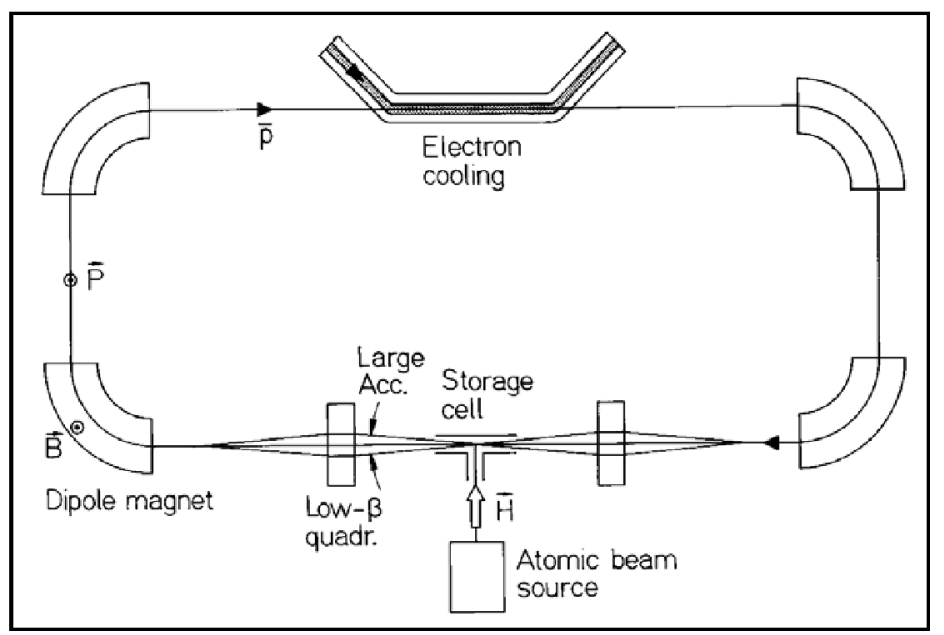

FIGURE 1. Principle of the Filter Experiment. See the text for an explanation.

at the target section provide a large angular acceptance. This way, the spinindependent losses by Coulomb scattering can be minimized. By measuring the leftright asymmetry of elastic protons, the beam polarization can be recorded continuously. The result of the test performed in 1992 is shown in Figure 2 [16].

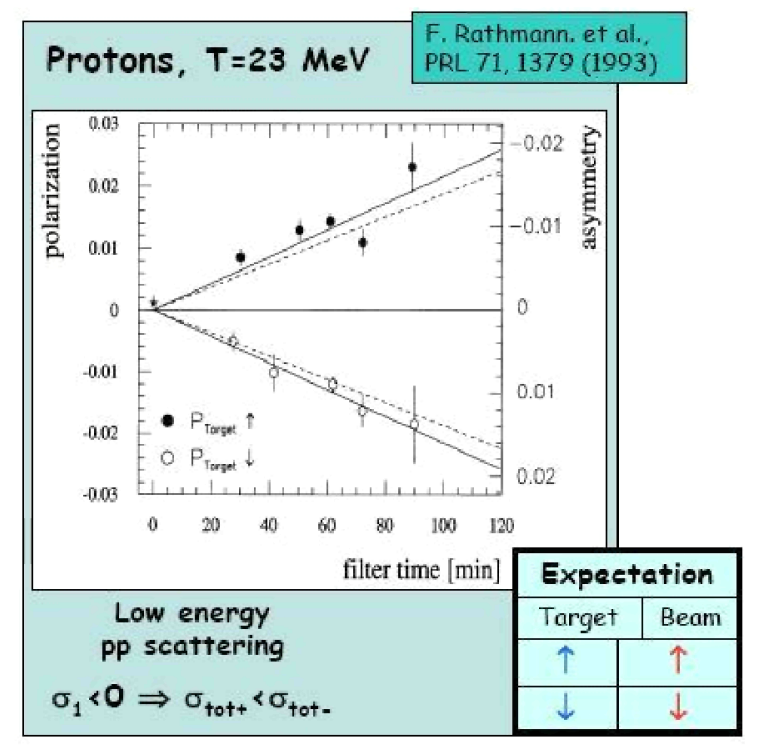

FIGURE 2. Result of the filter test for protons of $23 \mathrm{MeV}$. The beam polarization measured after a certain filter time is plotted for two orientations of the target polarization. The expected sign of the induced polarization is indicated in the insert and agrees with the data. 
A clear build-up of polarization is visible, correlated with target polarization up or down. This result represents the first and only result on polarization build-up of a stored ion beam. The measured rate $\mathrm{dP} / \mathrm{dt}=(1.24 \pm 0.06) \cdot 10^{-2} / \mathrm{h}$ was about half of the naive expectation [16] based on nuclear interaction. In 1994, Meyer [17] presented a quantitative explanation of the FILTEX result. Agreement with the data was obtained by including two more terms: 1 . spin transfer from polarized target electrons, and 2 . scattering of polarized target protons into the ring acceptance. As experimental opportunities were lacking, this explanation has not been questioned for a whole decade. With the advent of the FAIR project in 2003, antiproton polarization became a key issue. In 2005, the concept for the antiproton polarizer has been optimized [18] resulting in higher predicted $\bar{p}$ polarization. The electromagnetic spin-flip induced by the polarized target electrons calculated in [19], became the principal polarizing mechanism. According to the simulations, at an acceptance angle of $30 \mathrm{mrad}$ the optimum beam energy was found around $40 \mathrm{MeV}$, resulting in a beam polarization in excess of 0.3 after two beam life times, both for protons or antiprotons.

In 2005, new theoretical activities by a Novosibirsk group (Milstein \& Strakhovenko) and a Jülich group (Nikolaev and Pavlov) shed some doubt on the original Meyer-Horowitz approach. Both groups concluded that the build-up is entirely due to spin-dependent removal of beam particles by scattering to angles $\theta \geq$ $\theta_{\text {acc. }}$ In 2007, a new method was proposed by T. Walcher, H. Arenhövel et al. (Mainz) [20] with the promising feature of being virtually loss-free. The idea is to utilize spin transfer by free electrons to co-moving ions at very low relative velocity. The proposal is based on numerical calculations of this process by Arenhövel (cited in [20]) indicating that for attractive systems $p e^{-}$or $\bar{p} e^{+}$the corresponding cross section may be as large as $10^{13} \mathbf{b}=10^{-11} \mathrm{~cm}^{2}$, resulting in very short build-up times. Clearly, this contradiction needed to be resolved.

\section{WORKSHOP AT THE COCKCROFT INSTITUTE}

The workshop, entitled "Polarized Antiproton Beams - How?" was held at the Cockcroft Institute of Accelerator Science and Technology, Warrington, UK, from 29 to 31 August 2007 [9]. The workshop was attended by 28 participants from six countries. It was organized in topical sessions, followed by discussion periods and a workshop summary, presented by H.-O. Meyer (Indiana).

The workshop was opened with two papers by Buttimore and O'Brien (both Dublin) on the various processes which may be utilized in the polarizer, like channeling by bent crystals, polarization transfer and spin filtering with its evolution equation thus clarifying the different mechanisms. Nikolaev and Pavlov (Jülich) presented their study [21] on $\bar{p}$ spin filtering and the processes introduced by Meyer [17]. They concluded that due to the cancellation of terms the target electrons do not contribute to the polarization build-up. The resulting polarizing cross section is in fair agreement with the re-analyzed result of the FILTEX test. A similar study was presented by Strakhovenko (Novosibirsk) based on an earlier paper by the Novosibirsk group [22]. They also obtain reasonable agreement with the re-analyzed FILTEX data of 1992. By using the Paris potential, predictions for $\bar{p}$ spin filtering were made as well. 
A number of contributions were related to proposals of the original Bodega Bay list. Barber (DESY and Cockcroft Institute) discussed the status of the various Spin Splitter proposals based on Stern-Gerlach (SG) forces. He stressed the importance to include all known processes that influence particle motion and stability in a storage ring. Otherwise the spatial separation estimated in, possibly over-simplified models is washed out by these effects. In his view, none of the papers claiming that polarization either by transverse or longitudinal SG forces can be produced, do meet these requirements.

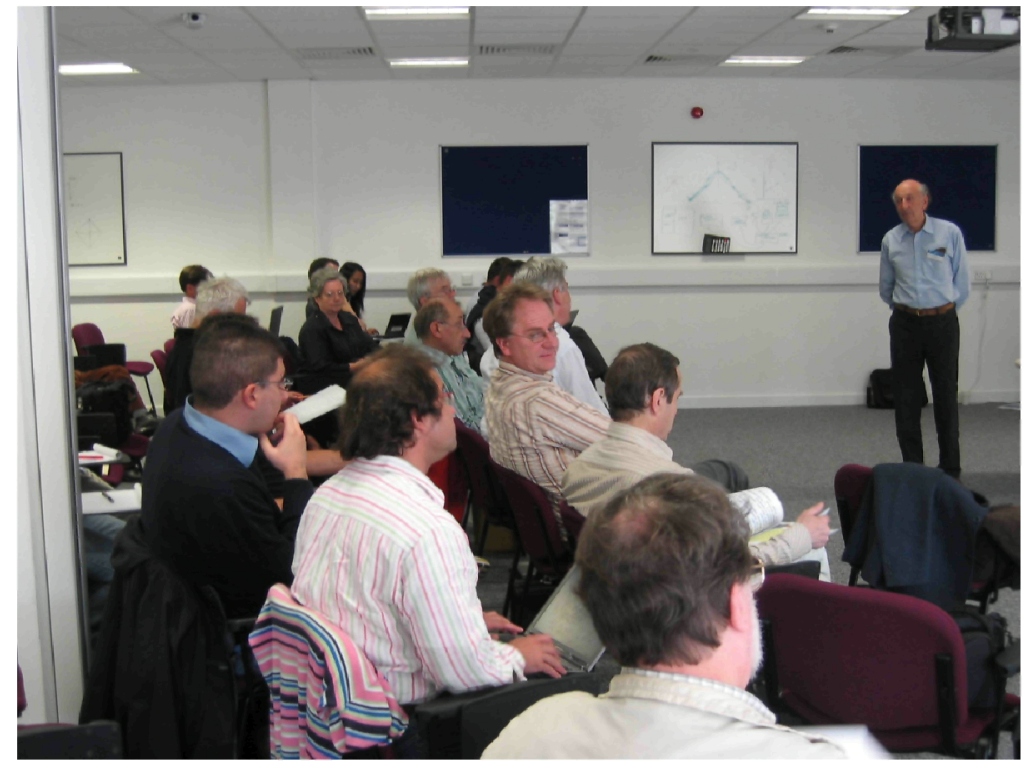

FIGURE 3. Participants of the Workshop at the Cockrcoft Institute during a discussion session, lead by E. Leader (London), right.

The idea of Dynamical Nuclear Polarization (DNP) "in flight" was presented by Krisch (Michigan), based on his results obtained with Jeffries and Kleppner at Bodega Bay. A beam of polarized electrons $\mathrm{e}^{ \pm}$overlaps with the stored $\bar{p}^{\prime} s$ at equal velocity in a strong magnetic field. By means of microwave radiation, lepton polarization is transferred to antiprotons, similar to the original DNP in solids, proposed by Jeffries in the 1950 's. According to their original estimates, the required $\mathrm{e}^{ \pm}$densities are still far beyond present day capabilities. Unless a special trick is found to reduce the effective e-p radius, as already stated 22 years earlier, this proposal is far from realization.

As shown by Lehrach (Jülich), storage rings as the stage for all these proposed methods are in good shape to provide the necessary features. The role of resonances and their crossing is deeply understood. Stable longitudinal polarization can be maintained by means of a Siberian Snake. The beam life time, in particular in the presence of strong cooling, is still an issue. For filtering studies as planned at COSY very long life times in the order of $10^{5} \mathrm{~s}$ are required. In such extreme situations, simulation studies both for the orbital and spin motion are of utmost importance. 
Barber illustrated in his talk on spin tracking the methods which allow one in principle to include stochastic effects like damping of betatron oscillations by cooling, or scattering, i.e. intra-beam scattering, or scattering on the residual gas or target gas. These effects need to be taken into account if spin filtering over long storage times is to be simulated reliably.

Polarization by channeling was an issue as well at Bodega Bay, stimulated by the Kaminsky experiments on low energy deuteron beams using magnetized thin $\mathrm{Ni}$ crystals [23]. Later, the observed polarization was traced back to the formation of fast $\mathrm{D}$ atoms by pick-up of polarized electrons from the solid, resulting in nuclear polarization due to re-coupling of electron and deuteron spin. The recent channeling proposal was inspired by the amazing results obtained on deflection of very high energy ions by means of bent single crystals as pioneered by the Protvino laboratory. Fiorini (Ferrara) gave an overview of similar experiments at the CERN SPS [24], illustrating the capability of deflecting $400 \mathrm{GeV}$ protons which follow the curvature of the bent Si single crystal. Ukhanov (Protvino) presented his proposal to utilize bent crystals as polarizer for energetic particle beams. It is well known that in elastic scattering to a particular side, e.g. to the left, the scattered particle has acquired some vector polarization perpendicular to the scattering plane. If many of these deflections to the same side occur as in a bent crystal, they should add up to some finite value. The total bending angle might be as large as $100 \mathrm{mrad}$ or more resulting in a high number of scattering processes of the order $10^{3}-10^{4}$. No measurements exist to date so that it is merely a matter of speculation which polarization to take per elementary scattering process. Experimental results of high energy elastic hadron scattering in the interference region were discussed by Buttimore, indicating rather unfavorable conditions for accumulating a significant polarization. Here experiments are urgently needed.

Two presentations dealt with electrons. Rathmann (Jülich) described an experiment foreseen for COSY to test the large cross sections for e-p polarization transfer at low relative velocity predicted by the Mainz group [20]. The idea is to store a polarized proton beam in COSY and detune the electron cooler periodically such that $v_{\text {rel }}$ in the e-p system is in the range for which the huge cross sections should occur. This would then result in fast depolarization of the protons. The sensitivity of this method was estimated to be better than $10^{-5}$ of the predicted cross section. The experiment has been performed in the meantime and is summarized at the end of this report. The $2^{\text {nd }}$ paper was also related to the Mainz proposal which requires a strong source for polarized positrons. Bailey (Cockcroft Inst., and Liverpool) described the concepts for sources of polarized positrons suitable for future $\mathrm{e}^{+} \mathrm{e}^{-}$colliders like ILC or CLIC. The technique of these sources would also be applicable within the new scheme to polarize $\bar{p}^{\prime} s$ by lepton spin transfer.

The workshop was summarized by Meyer (Indiana) with exceptional clarity. He divided the proposed methods into two groups. The three methods in limbo were Channeling, Stern-Gerlach Separation and Dynamic Nuclear Polarization in Flight. Significant theoretical efforts have been spent on these methods. A final judgment depends on experimental tests which are lacking in all these cases. The other group of proposals deals with a beam orbiting through a polarized target. The latter may consist of atoms or electrons. Meyer pointed out that (at the time of the workshop) two 
contradicting explanations of the FILTEX result existed, one by the JülichNovosibirsk groups that spin transfer by electrons plays no role, and the other including this effect. The depolarization test proposed for COSY has the potential to discriminate between these models und is therefore highly desirable.

\section{WE-HERAEUS SEMINAR AT BAD HONNEF}

The $409^{\text {th }}$ Wilhelm und Else Heraeus Seminar, entitled "Polarized Antiprotons", was held at the Physikzentrum of the DPG at Bad Honnef near Bonn from 22 to 25 June 2008, organized by P. Lenisa (Ferrara) and F. Rathmann (Jülich), see Figure 4. It was generously sponsored by the Wilhelm und Else Heraeus-Stiftung and attended by 57 participants. Out of 27 presentations, eight were about general spin physics theory and experiment and five on spin physics with polarized $\bar{p}^{\prime} s$. Four presentations dealt with theoretical aspects of polarizing $\bar{p}^{\prime} s$ and three with experimental ones. Six machine-related talks covered spin filtering and future machines for $\bar{p} \uparrow$ experiments. Available space does not permit to mention all speakers. The talks can be found at http://www.fe.infn.it/heraeus/talks/.

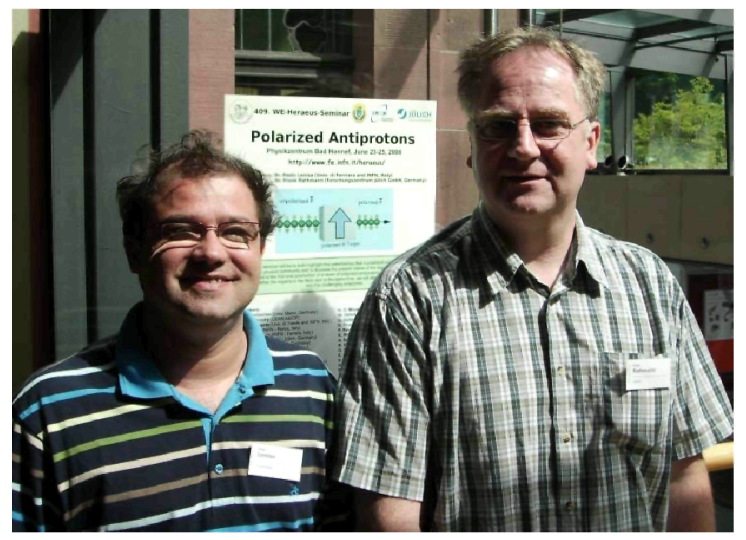

FIGURE 4. The organizers of the Heraeus Seminar, P. Lenisa and F. Rathmann, at the Physikzentrum during the workshop.

In the opening talk, Rith (Erlangen) gave an overview of experimental results in high energy spin physics. The lesson from the past is that new experiments are usually based on technological achievements. Polarized antiprotons have the potential of being a powerful new tool. In a number of talks, the status of experiments on nucleon spin structure like COMPASS or HERMES was discussed. Other topics were the potential of the Drell-Yan process for various measurements and its comparison to DIS, special aspects of $\bar{p}$ induced reactions and predictions from theory about spin observables, e.g. for $\bar{p} p$ interaction. The possibility to perform polarization experiments at PANDA detector at HESR were discussed by Maas (GSI and Mainz). An overview was given by Meyer (Indiana) on polarization experiments in storage rings, underlining the power of this technique. 


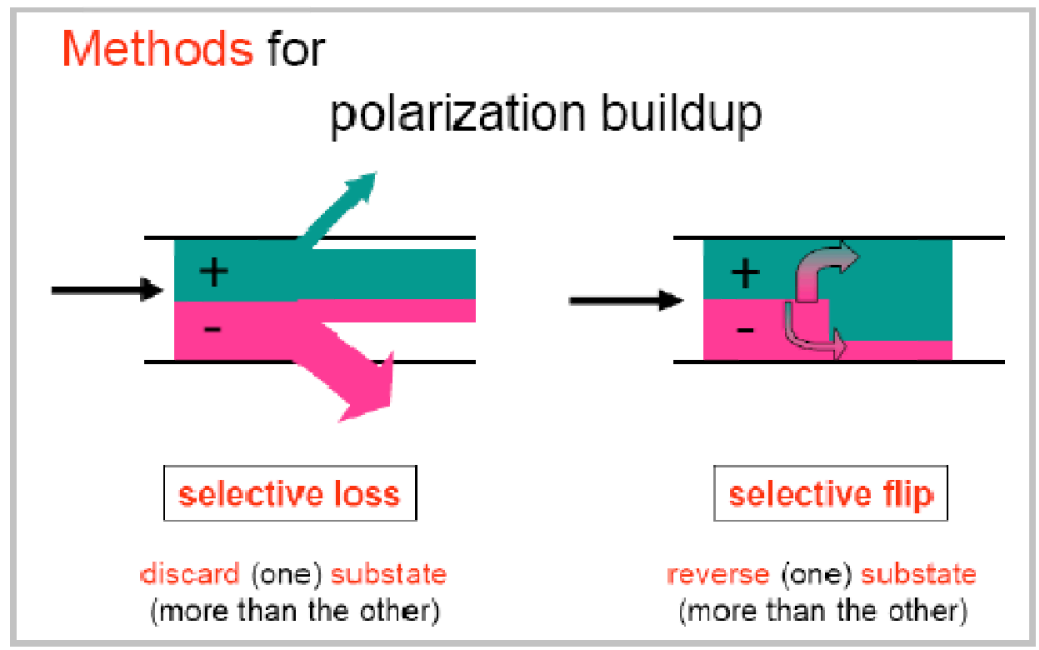

FIGURE 5. Classification of the two classes of mechanisms for a polarization build-up of a beam traversing a polarizer: selective loss (left) and selective spin flip (right). Taken from H.-O. Meyer's summary talk in [9]

The main topic at the Honnef meeting was what can be learned from the successful FILTEX test in 1992, and how the result can be understood quantitatively. The key question was how the polarization build-up works (see Fig. 5). The revised Mainz proposal was presented by Walcher (Mainz), based on the existence of electron spin transfer to the proton (or $\mathrm{e}^{+}$spin transfer to the antiproton). The alternative view was presented by Strakhovenko (Novosibirsk), concluding that this mechanism is by many orders of magnitude too low to contribute compared with selective removal. As mentioned at the Cockcroft workshop, a depolarization experiment was being prepared at COSY. The first result presented at the Heraeus Seminar by Oellers (Jülich) was eagerly awaited. It clearly indicated no significant depolarization of the stored protons by the electrons of the cooler which were periodically detuned to the right velocity difference predicted by the Mainz calculations. The sensitivity was such that a possible spin transfer cross section should be very small compared to the predicted $10^{13}$ barn. A more advanced status of the analysis has been presented at Spin 2008 by Rathmann [11]. The conclusion has not been changed confirming that the result of the Novosibirsk-Jülich groups is correct.

This result has important implications. The possibility to utilize an electromagnetic and thus calculable process as polarizer does not exist. Spin filtering based on the spin dependence of the - partly unknown - $\bar{p} p$ interaction seems to be the only way. This makes $p p$ filtering tests at COSY, and in particular $\bar{p} p$ filtering tests at the CERN AD indispensible mile stones on the way to polarized antiprotons!

In a number of presentations, the necessary ingredients for spin filter experiments were discussed:

1. Long polarization life time, which seems $\mathrm{OK}$ 
2. Long beam life time, in particular at low energies, e.g. at COSY injection energy, where the observed times are shorter than the naive expectation based on chamber limits

3. Polarized $\mathrm{H}$ and $\mathrm{D}$ targets with high density and polarization; more density could be used if available

4. A target section with large acceptance angle to minimize the (spinindependent) Coulomb losses

5. A target cell with sufficient opening during beam injection; this is particularly demanding at the $\mathrm{AD}$

6. Detector systems with large acceptance capable of standing the high rates involved

7. Siberian snakes to allow for longitudinal polarization at the target which gives a higher build-up rate

In his summary, Ströher (Jülich) presented the mile stones on the way towards polarized antiprotons at FAIR: (2010) measurement of proton spin filtering at COSY; (2011/12) measurement of antiproton spin filtering at AD; (2013) final design of antiproton polarizer, based on a preliminary design [5]. He reminded the community that NOW there is a unique chance. A clear roadmap and a strong commitment of the PAX collaboration exist. A NEW experimental result - sixteen years after the first one - has been achieved. Strong support of the community is needed in order to create a polarized antiproton facility at FAIR. Given the lively discussions at the Heraeus Seminar, and the many young and dedicated participants, the route to polarized antiprotons at FAIR seems accessible.

\section{CONCLUSIONS}

The lack of polarized antiprotons has been regarded as shortcoming of the LEAR program restricting the reach of what could be learnt about the spin dependence of the $\bar{p} p$ interaction. All attempts to produce polarized antiproton beams of significant quality were unsuccessful [25], or the results came too late to make an impact. The upcoming FAIR facility with its strong antiproton program has renewed the interest in antiproton polarization. This missing tool would enable exciting new physics as exemplified in the talk by Anselmino at this meeting [7] on the direct measurement of transversity of the nucleon in double-polarized Drell-Yan, or in a number of talks at the Heraeus Seminar [10], at this conference or elsewhere. Let us work together in order to add this missing, extremely powerful instrument to our tool box!

\section{ACKNOWLEDGMENTS}

I thank the german BMBF for financial support of the research grant 06ER144. Discussions with my colleagues, in particular with A. Kacharava, P. Lenisa, A. Nass, H.-O. Meyer, F. Rathmann, K. Rith and H. Ströher, are gratefully acknowledged. 


\section{REFERENCES}

1. E. Klempt, F. Bradamante, A. Martin, and J.M. Richard, Phys. Rep. 368 (2002) 119.

2. E. Steffens et al., in: Proc. $3^{\text {rd }}$ LEAR Workshop, Tignes, 1985, p.245; and CERN Proposal CERN/PSCC/85-80 (Nov. 5, 1985).

3. http://www.gsi.de/fair/index_e.html

4. http://www.fz-juelich.de/ikp/pax

5. A. Garishvili et al: Design Study for an Antiproton Polarizer Ring (APR). Proceed. EPAC 2006, Edinburgh (2006), MOPCH083.

6. http://www.fz-juelich.de/ikp/pax/proposals/files/techproposal20060125.pdf

7. M. Anselmino: Transverse Spin Structure of the Nucleon. These proceedings.

8. Proc. of the Workshop on Polarized Antiprotons, Bodega Bay, CA, 1985. A.D. Krisch, A.M.T. Lin, O. Chamberlain (Edts.), AIP Conf. Proc. 145 (1986) 207.

9. Proc. of the Int. Workshop "Polarized Antiproton Beams - How?", Cockcroft Institute, Daresbury, UK 29 - 31 August 2007. S. Chattopadhyay, D.P. Barber, N. Buttimore, G. Court, E. Steffens (Edts.), AIP Conf. Proc. 1008 (2008).

10. http://www.fe.infn.it/heraeus/

11. F. Rathmann: Do unpolarized electrons affect the polarization of a stored proton beam? These proceedings.

12. F.L. Shapiro: Nuclear Structure Studies with Neutrons. Proc. Int. Conf. Study of Nucl. Structure with Neutrons, Antwerp 1965, North-Holland, Amsterdam (1966), p. 588.

13. T.R. Gentile et al, Physica B 356 (2005) 96.

14. T.O. Niinikoski and R. Rossmanith, NIM A 255 (1987) 460.

15. D. Habs et al, Nucl. Instr. Meth. B 43 (1989) 390.

16. F. Rathmann et al., Phys. Rev. Lett. 71 (1993) 1379.

17. H.O. Meyer, Phys. Rev. E 50 (1994) 1485.

18. F. Rathmann et al, Phys. Rev. Lett. 94 (2005) 014801.

19. C.J. Horowitz and H.O. Meyer, Phys. Rev. Lett. 72 (1984) 3982.

20. T. Walcher et al, Eur. Phys. J. A 34 (2007) 447.

21. N. Nikolaev and F. Pavlov, arXiv:0701175v1 [hep-ph] (2005).

22. A.I. Milstein, V.M. Strakhovenko, Phys. Rev. E 72 (2005) 066503.

23. M. Kaminsky, Phys. Rev. Lett. 23 (1969) 819.

24. W. Scandale et al., Phys. Rev. Lett. 98 (2007) 154801.

25. A. Martin et al., Nucl. Phys. A 487 (1988) 563 